\title{
Influência de ventilação e aspersão em sistemas de sombreamento artificial para vacas em lactação em condições de calor
}

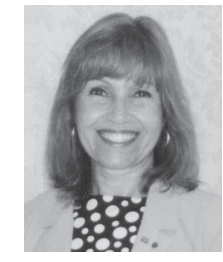

Irenilza de A. Nääs ${ }^{1}$ \& Irineu Arcaro Júnior ${ }^{2}$

\author{
1 Faculdade de Engenharia Agrícola da UNICAMP, C.P. 6011, CEP 13081-970, Campinas, SP. Fone: (19) 3788-1039. \\ E-mail: irenilza@agr.unicamp.br (Foto) \\ 2 Instituto de Zootecnia. Nova Odessa, SP. E-mail: iarcaro@izsp.br
}

Protocolo $109-30 / 08 / 2000$

\begin{abstract}
Resumo: O presente trabalho foi desenvolvido na Estação Central do Instituto de Zootecnia, localizada em Nova Odessa, SP, e teve por objetivo avaliar três diferentes métodos físicos destinados a promover a redução do estresse térmico em vacas em lactação. Os tratamentos testados foram: sombras artificiais produzidas por tela de propileno $80 \%$; sombras idênticas às anteriores, acrescidas de ventilação, e sombras combinando ventilação e aspersão. Utilizaram-se 18 fêmeas bovinas distribuídas ao acaso dentro dos tratamentos. O experimento teve a duração de 180 dias, sendo realizado durante o período de outono. Os parâmetros avaliados foram: produção e composição do leite, temperatura retal, freqüência respiratória, freqüência cardíaca e parâmetros meteorológicos do ambiente. Os valores da produção de leite apresentaram diferenças significativas, sendo que o tratamento sombra + ventilação + aspersão, apresentou a melhor média $(20,53 \mathrm{~kg})$, seguido dos sombra + ventilação $(19,19 \mathrm{~kg})$ e sombra $(18,20 \mathrm{~kg})$. Pela avaliação dos dados ficaram evidentes os benefícios do uso de sombras artificiais e dos sistemas de ventilação e aspersão.
\end{abstract}

Palavras-chave: produção de leite, ambiência, sombreamento

\section{Influence of the ventilation and sprinkling in artificial shading system for dairy cows in hot climate}

\begin{abstract}
The present research was carried out at Animal Science Institute, in São Paulo and had the objective of evaluating three different types of cooling systems (physical) to reduce thermal stress for milking cows. The tested treatments used artificial shade using a propylene screen $80 \%$ direct solar radiation reduction; the same shade using a $0.5 \mathrm{HP}$ fan; and the same shade structure using a $0.5 \mathrm{HP}$ fan with fogging system. Eighteen milking cows were used in the treatments - 12 Holstein cows and 6 brown swiss - equally distributed in the treatments according to their age and milk production level. The parameters analyzed were: milk production and fat content, body temperature, respiratory frequency, and cardiac frequency. Environmental parameters measured were: dry bulb, black globe temperature and relative humidity, collected in each treatment and at a meteorological station at a nearby site. The results showed that the best treatment was the shade using forced ventilation associated with a fogging system.
\end{abstract}

Key words: milk production, environment, shading

\section{INTRODUÇÃO}

Cerca de dois terços do território brasileiro situa-se na faixa tropical do planeta, onde predominam as altas temperaturas do ar, conseqüência da elevada radiação solar incidente.

A vaca leiteira é um animal homeotérmico, com temperatura interna de $38,5^{\circ} \mathrm{C}$, freqüência cardíaca de 60 a 80 pulsações por minuto e 10 a 30 movimentos por minuto de freqüência respiratória. Esta temperatura sofre pequena variação durante o dia, sendo mais alta no final das tardes e no início da noite, variando também durante o ciclo estral e entre as estações do ano (Head, 1995).

Nääs (1989) afirmou ser a faixa de 13 a $18^{\circ} \mathrm{C}$ confortável para a maioria dos ruminantes, e referiu-se às temperaturas entre $4 \mathrm{e}$
$24{ }^{\circ} \mathrm{C}$ para vacas em lactação, podendo ser restringidas essas faixas aos limites de 7 a $21^{\circ} \mathrm{C}$, em função da umidade relativa e da radiação solar.

Como resposta ao estresse térmico, os animais reagem com mudanças fisiológicas e comportamentais (Baêta et al., 1998). As respostas fisiológicas são: aumento da freqüência respiratória que, em geral, apresenta-se superior a 40 movimentos respiratórios por minuto; aumento da freqüência cardíaca; redução na ingestão de alimentos e aumento na ingestão de água. O consumo de alimentos é o principal fator responsável pelo decréscimo na produção de leite em condições de estresse (Huber, 1995). 
Head (1989), estudando vacas holandesas sob altas temperaturas e altas umidades relativas, afirmou que o estresse térmico causou decréscimos na produção e no teor de constituintes do leite, como a gordura, proteína, ácido cítrico, cálcio e potássio; já Rodrigues et al. (1985) trabalhando com vacas holandesas em produção, submetidas a estresse térmico de inverno ameno e verão, encontraram valores de gordura de 3,85 e $3,31 \%$, e de proteína de 3,42 e $2,98 \%$, quando a temperatura foi de 8 e $32^{\circ} \mathrm{C}$, respectivamente.

Roman-Ponce et al. (1977) concluíram que, em região de clima subtropical, vacas alojadas em abrigos sombreados contra a radiação solar, produziram 10,7\% mais leite e apresentaram maior taxa de concepção, quando comparadas com aquelas alojadas em locais sem proteção. As perdas de calor realizadas pela vaca através da superfície da pele e do trato respiratório não são suficientes em climas quentes e úmidos, para a ótima produção de leite (Bucklin et al. 1998).

Até um limite aproximado de umidade relativa do ar de $70 \%$, a melhor maneira de se resfriar um ambiente destinado a animais em lactação, é fazer uso da água, uma vez que a mesma possui alta capacidade calorífica e elevado calor latente de vaporização. Um outro sistema que pode ser empregado quando a temperatura ambiente for superior à ótima, consiste em se aumentar a taxa de ventilação, através do acréscimo da taxa de renovação do ar favorecendo a perda de calor por via convectiva, conseqüentemente eliminando o calor produzido pelos animais, para evitar temperatura excessiva dentro da instalação.

Em pesquisa realizada no Missouri, com vacas holandesas submetidas a aspersão de água das 11:00 às 17:30 h, à sombra, nos dias em que a temperatura do ar excedeu $27^{\circ} \mathrm{C}$, produziram $700 \mathrm{~g}$ a mais de leite por dia que suas companheiras sem acesso à aspersão (Igono et al., 1985).

$\mathrm{Na}$ Austrália subtropical, vacas holandesas de alta produção, confinadas e com livre acesso à sombra e forragem, recebendo concentrado na base de $9 \mathrm{~kg} \mathrm{vaca}^{-1} \mathrm{~d}^{-1}$ e aspergidas com água sempre que a temperatura do ar excedeu $26{ }^{\circ} \mathrm{C}$, produziram $4,8 \mathrm{~kg} \mathrm{~d}^{-1}$ a mais de leite que seus pares sem aspersão de água (Hall et al., 1997).

Igono et al. (1985) utilizaram aspersão de água em vacas holandesas durante o verão, no Estado de Colúmbia (EUA) e encontraram acréscimos de $0,7 \mathrm{~kg}$ de leite quando as vacas foram comparadas com as sem aspersão. $\mathrm{O}$ efeito da aspersão foi evidente mesmo em verão moderado, onde o ITU (índice de temperatura e umidade) foi ligeiramente superior ao ponto onde a produção de leite começa a decrescer. Em trabalhos realizados com vacas em lactação nos Emirados Árabes, segundo Igono et al. (1985), as perdas de produção de leite, devido ao verão, foram reduzidas em $2,8 \%$, com o uso de aspersão de água, sendo que, no total de 305 dias de lactação, representaram $0,6 \%$.

Frazzi et al. (1997), trabalhando com vacas holandesas alojadas em instalação parcialmente fechada, localizada no Vale do Pó, Itália, concluíram que ventilação na velocidade de $0,5 \mathrm{~m} \mathrm{~s}^{-1}$ reduziu os efeitos negativos do estresse térmico. As vacas que receberam ventilação, comparadas com o controle, apresentaram menor aumento na temperatura retal $\left(39,49 \times 38,94^{\circ} \mathrm{C}\right)$, na taxa respiratória e menor redução na produção de leite. Os autores observaram ainda que, quando além da ventilação se utilizou a aspersão, as vacas não apresentaram aumento na temperatura retal, registrando-se pequenas diminuições na taxa respiratória. Relatam aumentos de 10 a $15 \%$ na umidade relativa e redução de 2,5 a $3{ }^{\circ} \mathrm{C}$ na temperatura ambiente, concluindo que o uso de ventiladores e aspersores, quando comparados com ventiladores, reduz o estresse térmico em gado leiteiro.
Fuquay et al. (1997) testaram o uso de ventilação em vacas lactantes em um clima onde a temperatura variou de 24 a $33{ }^{\circ} \mathrm{C}$ e a umidade relativa de 70 a $90 \%$. Os maiores valores de temperatura retal foram encontrados para as vacas sem ventilação, não havendo diferença na produção de leite, sendo que as vacas do grupo controle apresentaram menor persistência da lactação. Folman et al. (1979) registraram, em experimento similar, diferenças na produção de leite e temperaturas retais e, também, perda de peso nos animais do grupo controle, o que pode ser atribuído à perda de apetite e concluíram, ainda, que estruturas de sombreamento suplementadas com ventiladores melhoraram as respostas produtivas em vacas lactantes.

Frazzi (1998) citado por Silva (1998) trabalhando com quatro sistemas de controle ambiental, onde se utilizou ventilação natural, ventilação forçada, ventiladores com aspersão de água e resfriamento com água fria, encontrou reduções na produção de leite de $11,9,8$ e $3,7 \%$, respectivamente.

O objetivo deste trabalho foi avaliar o efeito de dois diferentes sistemas de acondicionamento de ambiente na redução do estresse térmico em vacas em lactação alojadas em piquetes com acesso à sombra artificial e, conseqüentemente, os seus efeitos sobre o desempenho produtivo desses animais.

\section{MATERIAL E MÉTODOS}

O experimento foi conduzido na Estação Experimental de Nova Odessa, no Instituto de Zootecnia da Secretaria da Agricultura do Estado de São Paulo, situada a $22^{\circ} 42^{\prime}$ de latitude Sul, $47^{\circ} 18^{\prime}$ de longitude Oeste e $550 \mathrm{~m}$ de altitude, no período de 1 de maio a 31 de outubro de 1999, coincidindo com o período mais seco do ano, apresentando valores altos de variação entre as temperaturas máximas e mínimas.

Foram utilizadas 18 fêmeas bovinas entre as $2^{\mathrm{a}} \mathrm{e} 4^{\mathrm{a}}$ lactações, estando no início do experimento entre 40 a 60 dias de paridas e apresentando produção de leite em torno de $25 \mathrm{~kg} \mathrm{dia}^{-1}$. O experimento foi inteiramente casualizado e para as avaliações do desempenho foram adotados os seguintes tratamentos: 1) sombras - S: tela protetora com vedação de $80 \%$ na quantidade de $5 \mathrm{~m}^{2}$ de área sombreada por animal, 2) sombras + ventilação - SV: sombras, acrescido de ventilação fornecida por ventilador axial, que era automatizado com um termostato para ligar quando a temperatura de bulbo seco fosse superior a $25^{\circ} \mathrm{C}$; e 3) sombras + ventilação + aspersão - SVA: sombras, a ventilação e aspersão de água fornecidas por um único aparelho, que era acionado por um termostato que, por sua vez, ligava o sistema quando a temperatura de bulbo seco ultrapassasse $25^{\circ} \mathrm{C}$.

Os parâmetros meteorológicos foram coletados em um abrigo contendo termômetro de bulbo seco, bulbo úmido, e termômetros de máxima e mínima, todos da marca Incotherm ${ }^{\circledR}$ a umidade relativa do ar foi determinada com higrômetro digital de marca Instrutherm ${ }^{\circledR}$. Esses dados foram obtidos na parte da manhã, às 8:00 e 11:00 he, na parte da tarde, às 13:00 e 16:00 h. Os animais permaneceram em piquetes de grama Estrela com a dimensão de $500 \mathrm{~m}$, providos de sombra, cocho e bebedouro. Receberam, como volumoso, silagem de milho e um concentrado à base de farelo de soja, grão de milho moído e minerais, que foram fornecidos na forma de dieta completa, duas vezes ao dia, de maneira a atender as exigências nutricionais para a manutenção e produção de leite, segundo o NRC.

Os animais, 6 por tratamento, foram ordenhados duas vezes ao dia, às 7:00 e às 16:00 $\mathrm{h}$, e a produção registrada diariamente. Semanalmente foi retirada amostra para determinação de 
gordura do leite. Os parâmetros avaliados nos diferentes tratamentos foram: produção, composição do leite, temperatura retal, freqüência cardíaca e freqüência respiratória. Essas medidas foram realizadas sempre antes das ordenhas e de maneira a não provocar movimentação excessiva dos animais.

Para os parâmetros produção de leite e porcentagem de gordura no leite, foi empregado o delineamento inteiramente casualizado, com três tratamentos (S, SV e SVA) e seis repetições (animais). Para os parâmetros freqüência cardíaca (FC), freqüência respiratória (FR) e temperatura retal (TR) utilizou-se o delineamento inteiramente casualizado, com três tratamentos (S, SV e SVA) e seis repetições (animais).

\section{RESULTADOS E DISCUSSÃO}

Na Tabela 1 são mostrados os valores de $\mathrm{F}$ referentes aos tratamentos dos dados fisiológicos, para o período da manhã.

Tabela 1. Valores médios* dos parâmetros fisiológicos freqüência respiratória (FR), freqüência cardíaca (FC) e temperatura retal (TR) dos animais para o período da manhã sob diferentes ambientes $(\alpha=1$ e $5 \%)$

\begin{tabular}{cccc}
\hline Fatores & \multicolumn{3}{c}{ Parâmetros Fisiológicos } \\
\cline { 2 - 4 } Ambiente & FR (99\%) & FC $(99 \%)$ & TR (95\%) \\
\hline S & $34,48 \mathrm{a}$ & $68,02 \mathrm{c}$ & $38,07 \mathrm{a}$ \\
SV & $32,56 \mathrm{~b}$ & $70,45 \mathrm{~b}$ & $38,72 \mathrm{a}$ \\
SVA & $34,76 \mathrm{a}$ & $75,25 \mathrm{a}$ & $37,09 \mathrm{a}$ \\
\hline \multicolumn{4}{c}{ Valores de F } \\
\hline Ambiente & $10,99^{\mathrm{S}}$ & $99,89^{\mathrm{S}}$ & $1,09^{\mathrm{NS}}$ \\
C V (\%) & 24,46 & 11,87 & 20,90 \\
\hline
\end{tabular}

"Médias seguidas pelas letras distintas na mesma coluna diferem entre si. NS = não significativo e $\mathrm{S}=$ significativo

Como os equipamentos se mantiveram desligados até que a temperatura ambiente alcançasse níveis acima de $25{ }^{\circ} \mathrm{C}$, quando se dava o acionamento automático dos sistemas, o que deve ter acontecido por volta das $10 \mathrm{~h}$, esta análise se refere ao efeito sombra artificial, muito mais que ao efeito sombra + equipamento. Tal situação se inverte nas horas relativas às medidas da tarde.

A freqüência respiratória teve um comportamento muito semelhante entre os tratamentos, diferindo apenas no tratamento S.

Com relação à freqüência cardíaca, os melhores resultados foram obtidos no tratamento SV, seguido do SV e do SVA. Quanto à temperatura retal, não houve diferença entre os tratamentos; entretanto, em valores absolutos, a média no tratamento correspondente a SVA possibilitou redução de 1,63 ${ }^{\circ} \mathrm{C}$ em relação ao tratamento $\mathrm{SV}$ e de $0,98{ }^{\circ} \mathrm{C}$ em relação ao tratamento S, semelhantes aos obtidos por Bucklin et al. (1998) e apresentaram reduções de 0,4 e $0,5{ }^{\circ} \mathrm{C}$ quando as vacas passaram por resfriamento.

$\mathrm{Na}$ Tabela 2 encontram-se os valores de F referentes aos tratamentos em relação aos dados fisiológicos, para o período da tarde.

No tratamento SV, a freqüência respiratória teve melhor resultado, seguida pelo tratamento SVA e, depois, pelo S. Com relação à freqüência cardíaca, os melhores resultados foram obtidos no tratamento S, seguido do SV e do SVA. Esta situação também se apresentou no período da manhã e supõe-se que se deve ao fato dos equipamentos apresentarem alto nível de ruído, quando ligados.
Tabela 2. Valores médios* dos parâmetros fisiológicos freqüência respiratória (FR), freqüência cardíaca (FC) e temperatura retal (TR) dos animais para o período da tarde sob diferentes ambientes ( $\alpha=1$ e $5 \%)$

\begin{tabular}{cccc}
\hline \multirow{2}{*}{$\begin{array}{c}\text { Fatores } \\
\text { Ambiente }\end{array}$} & FR $(99 \%)$ & FC $(95 \%)$ & TR $(95 \%)$ \\
\cline { 2 - 4 } S & $47,61 \mathrm{c}$ & $80,46 \mathrm{c}$ & $38,52 \mathrm{a}$ \\
SV & $40,38 \mathrm{a}$ & $81,68 \mathrm{~b}$ & $39,01 \mathrm{a}$ \\
SVA & $42,78 \mathrm{~b}$ & $88,44 \mathrm{a}$ & $38,59 \mathrm{a}$ \\
\hline \multicolumn{4}{c}{ Valores de F } \\
\hline Ambiente & $46,03^{\mathrm{S}}$ & $116,88^{\mathrm{S}}$ & $0,60^{\mathrm{NS}}$ \\
CV $(\%)$ & 28,97 & 10,93 & 19,97 \\
\hline
\end{tabular}

" Médias seguidas pelas letras distintas na mesma coluna diferem entre si. NS = não significativo e $\mathrm{S}=$ significativo

A temperatura retal média obtida nos três tratamentos não apresentou diferença significativa. Quanto à produção de leite, os resultados estão sumarizados na Tabela 3.

Tabela 3. Valores médios* de produção de leite nos períodos de manhã e tarde, e total diário, para os tratamentos S, SV e SVA $(\alpha=1 \%)$

\begin{tabular}{cccc}
\hline \multirow{2}{*}{ Fatores } & \multicolumn{3}{c}{ Parâmetros } \\
\cline { 2 - 4 } Ambiente & Manhã & Tarde & Total \\
\hline S & $11,71 \mathrm{c}$ & $6,44 \mathrm{~b}$ & $18,20 \mathrm{c}$ \\
SV & $12,48 \mathrm{~b}$ & $6,71 \mathrm{a}$ & $19,19 \mathrm{~b}$ \\
SVA & $13,74 \mathrm{a}$ & $6,78 \mathrm{a}$ & $20,53 \mathrm{a}$ \\
\hline \multicolumn{4}{c}{ Valores de F } \\
\hline Ambientes & $346,27^{\mathrm{s}}$ & $23,97^{\mathrm{s}}$ & $174,00^{\mathrm{S}}$ \\
$\mathrm{CV}(\%)$ & 14,49 & 18,22 & 15,20 \\
\hline
\end{tabular}

"Médias seguidas pelas letras distintas diferem entre si; $\mathrm{S}=$ significativo

A produção média de leite total no período da manhã foi significativamente maior para as vacas que receberam o tratamento SVA, seguida do tratamento SV e do tratamento S. No período da tarde os tratamentos SVA e SV não apresentaram diferenças estatísticas, embora o tratamento SVA tenha apresentado maior média. Quando se comparam os tratamentos SVA e SV com o tratamento S, existem diferenças significativas, evidenciando os benefícios proporcionados pelos equipamentos.

Ao se analisar a produção de leite total do dia, nota-se que os dados relativos ao tratamento SVA apresentaram maior média, que diferiu estatisticamente dos tratamentos SV e S, sendo que o tratamento SV foi melhor que o tratamento S. Em números absolutos, as vacas submetidas ao tratamento SVA obtiveram média de produção de leite de $12 \%$ superior ao tratamento $\mathrm{S}$, e as do tratamento $\mathrm{SV}$ foram $5 \%$ superiores ao tratamento $\mathrm{S}$, porém ao se comparar o tratamento SVA com o $\mathrm{SV}_{1}$, os valores absolutos indicam acréscimo de 6\%. Esses valores estão de acordo com os citados por Bucklin et al. (1998) que encontraram valores $11,6 \%$ maiores em vacas recebendo resfriamento através de aspersores e apresentando produções de leite por dia de 18,1 e $20,2 \mathrm{~kg}$, semelhantes àquelas encontradas. O mesmo autor afirma que a utilização de aspersão e ventilação, associadas a sombras, vêm sendo largamente utilizadas e têm mostrado aumento de 10 a $25 \%$ em comparação com as vacas que recebem somente sombras.

Resultados semelhantes foram encontrados por Hall et al. (1997) em que as vacas que recebiam aspersão de água sempre 
que a temperatura atingiu $26^{\circ} \mathrm{C}$, apresentaram produção de 4,8 kg a mais, quando comparadas com as sem aspersão; esses dados são semelhantes aos mencionados por Frazzi et al. (1998) que encontraram reduções na produção de leite, de 11, 9, 8 e $3,7 \%$, quando os animais foram submetidos a ventilação natural, ventilação forçada, ventiladores com aspersão de água e resfriamento com água fria. Igono et al. (1985) encontraram acréscimo de $0,7 \mathrm{~kg}$ de leite quando vacas receberam aspersão com água, quando comparadas com as sem água.

A Tabela 4 mostra os valores médios de percentagem de gordura para os diversos tratamentos.

Tabela 4. Valores médios de percentagem de gordura para os tratamentos $(\alpha=5 \%)$

\begin{tabular}{|c|c|}
\hline Fatores Ambiente & $\%$ Gordura \\
\hline $\mathrm{S}$ & $4,08 \mathrm{a}$ \\
\hline SV & $4,04 \mathrm{a}$ \\
\hline SVA & $4,17 \mathrm{a}$ \\
\hline \multicolumn{2}{|c|}{ Valor de F } \\
\hline Ambientes & $1,68^{\mathrm{NS}}$ \\
\hline CV $(\%)$ & 12,91 \\
\hline
\end{tabular}

Não houve diferença significativa entre os tratamentos; esses valores diferem dos resultados encontrados por Head (1989) e por Rodrigues et al. (1985) que afirmam que altas temperaturas provocam quedas na produção de gordura e componentes do leite. Os valores médios de percentagem de gordura obtidos nesse experimento, foram, para todos os tratamentos, maiores que os obtidos por esses autores. Considerando-se que, segundo estes, altas temperaturas provocam queda na produção de gordura do leite, espera-se que os três sistemas de acondicionamento térmico tenham sido benéficos no arrefecimento e contribuído para o conforto das vacas.

\section{CONCLUSÕES}

1. Embora tenha havido diferenças estatísticas com relação a alguns parâmetros fisiológicos entre os tratamentos, estas foram não conclusivas, provavelmente devido ao pequeno número de repetições (vacas por tratamento) utilizado no experimento.

2. As vacas alojadas no tratamento SVA demonstraram ter sido submetidas ao melhor sistema de arrefecimento térmico, uma vez que apresentaram melhores resultados de desempenho produtivo.

3. O tratamento SVA apresentou melhor resultado absoluto nos níveis de percentagem de gordura. Dentre os sistemas estudados, o tratamento contendo sombra artificial associado a ventilação mais nebulização foi o melhor sendo, portanto, recomendado para vacas em lactação alojadas em piquetes.

\section{LITERATURA CITADA}

Baêta, F.C. Instalações para gado leiteiro na região do mercosul. In: Congresso Brasileiro de Biometeorologia, 2. Anais... SBB. Goiânia, 1998, p.162-173.
Bucklin, R.A.; Bray, D.R. The american experience in dairy management in warm and hot climates. In: Simpósio Brasileiro de Ambiência na Produção de Leite, 1. Anais... 1998, p.156-174.

Folman,Y.; Berman, A.; Hertz, Z.; Kaim, M.; Rosemberg, M.; Mamen, M.; Gordin, S. Milk yield and fertility of high-yielding dairy cows in a subtropical climate during summer and winter. Journal of Dairy Research, Iowa, 1979, v.46, p.411-425.

Frazzi, E.; Calamari, L.; Calegari, F. Different systems of air conditioning for dairy cows housing in Mediterranean climate. In: International Congress on Agricultural Engineering, $13^{\circ}$. Marrocos, 1998, p.23-29.

Frazzi, E.; Calamari, L.; Calegari, F.; Maianti, M.G.; Cappa, V. The aeration, with and without misting: Effects on heat stress in dairy cows. Proceeding of International Symposium, 5, Minnesota, 1997, p.907-914.

Fuquay, J.W. Heat stress as it affects animal production. Livestock Environment, V, Iowa, v.2, p.1133-1137. 1997.

Hall, A.B.; Young, B.A.; Goodwin, P.J.; Gaughan, J.M.; Davison, T. Alleviation of excessive heat load in the high producing dairy cow. Livestock Environment, V, Iowa, v.2, p.928-935. 1997.

Head, H.H. The strategic use of the physiological potential of the dairy cow. In: Simpósio Leite nos Trópicos: Novas estratégias de produção. Anais... Botucatu, 1989. p.38-89.

Head, H.H. Management of dairy cattle in tropical and subtropical environments. In: Congresso Brasileiro de Biometerologia, 2, Anais... SBBiomet, Jaboticabal, 1995, p.26-68.

Huber, H. Manejo de animais em sistema de estabulação livre visando maximizar o conforto e a produção. In: Congresso Brasileiro de Gado Leiteiro, 2, Anais... Piracicaba 1995, p.41-68.

Igono, M.O.; Steevens, B.J.; Shanklin, M.D.; Johson, H.D. Spray cooling effects on milk production, milk and rectal temperature of cows during a moderate temperature summer season. Journal of Dairy Science, Wisconsin, v.68, p.979-985. 1985.

Nääs, I. de A. Princípios de conforto térmico na produção animal. 1 ed. São Paulo: Icone Editora Ltda., 1989, 183p.

Rodrigues, L.A.; McKonnen, G.; Wilcox, C.J.; Martin, F.G.; Krienke, W. A. Effects of relative humidity, maximum and minimum temperature, pregnancy, and stage of lactation on milk composition and yield. Journal of Dairy Science, Wisconsin, v.68, p.973-978,1985.

Roman-Ponce, H.; Thatcher, W.W.; Buffington, D.E.; Wilcox, C.J.; van Horn, H.H. Physiological and production responses of dairy cattle to a shade structure in a subtropical environment. Journal of Dairy Science, Wisconsin, 1977, v.60, p.424-430.

Silva, I.J.O. Climatização das instalações para bovinos leiteiros. In: Simpósio Brasileiro de Ambiência na Produção de Leite, 1. Anais... 1998, p.114-145. 